\title{
Theory of Hypernumbers and Extrafunctions: Functional Spaces and Differentiation
}

\author{
MARK BURGIN \\ Department of Mathematics, University of California, Los Angeles, 405 Hilgard Ave., Los Angeles, CA 90095, USA
}

(Received 15 August 2001)

\begin{abstract}
The theory of hypernumbers and extrafunctions is a novel approach in functional analysis aimed at problems of mathematical and computational physics. The new technique allows operations with divergent integrals and series and makes it possible to distinct different kinds of convergence and divergence. Although, it resembles nonstandard analysis, there are several distinctions between these theories. For example, while nonstandard analysis changes spaces of real and complex numbers by injecting into them infinitely small numbers and other nonstandard entities, the theory of extrafunctions does not change the inner structure of spaces of real and complex numbers, but adds to them infinitely big and oscillating numbers as external objects. In this paper, we consider a simplified version of hypernumbers, but a more general version of extrafunctions and their extraderivatives in comparison with previous works.
\end{abstract}

Keywords: Functional analysis; Differentiation; Extraderivative; Extrafunction; Hypernumber; Distribution

\section{INTRODUCTION}

The theory of hypernumbers and extrafunctions emanated from physically directed thinking and was derived by a natural extension of the classical approach to the real number universe construction. Namely, an important class of problems that appear in contemporary physics and involve infinite values inspired this theory. As it is known, many mathematical models, which are used in modern theories of elementary particles (such as gauge theories) and some other physical theories, imply divergence of analytically calculated properties of physical systems. The simplest example is the case of a free electron when its interaction with photons changes the energy of the electron so that the energy becomes infinite (in a model). Mathematical investigation of many physical problems gives rise to divergent integrals and series that are such mathematical constructions that have, in some sense, infinite values. However, physical measurements give, as the result, only finite values. That is why, many methods of divergence elimination (regularization), i.e. of elimination of infinity, have been elaborated (cf., for example, Edzawa and Zuneto 1977 or Collins, 1984). Nevertheless, the majority of them were not well grounded mathematically because they utilized operations with formal expressions that had neither mathematical nor physical meaning. Moreover, there are such models in physics that contain infinities that cannot be eliminated by these methods based on existing mathematical theories. Only in the theory of hyperintegration (Burgin, 1990; 1995a), based on the theory of hypernumbers, all divergent integrals and series that appear in the calculations with physical quantities become correctly grounded as strict mathematical objects.

In addition to this, theory of hyperintegration suggests a new approach to functional (path) integrals (Burgin, 2000b). An important peculiarity of this approach is that functional integrals are treated as ordinary integrals in which hypermeasures are used instead of ordinary measures. Moreover, it is possible to apply this approach to develop an integral calculus for arbitrary functional spaces.

The new theory provides also new facilities for mathematics and numerical computations. For example, there was time when mathematicians (such as Leonard Euler, who was the greatest mathematician of his time) manipulated with divergent series (that have infinite values) in the same way as they treated convergent series (that have finite values). But it was demonstrated (in the context of real and complex numbers) that such manipulations were not mathematically correct and led to contradictions. Utilization of asymptotic series, which are divergent, for function approximation (cf., for example, Collins, 1984) is another example of a similar 
situation. Transition to hypernumbers provides correct mathematical means to deal with such constructions in a proper way.

This approach correlates with problems of modern physical theories in which physical systems are described by chaotic processes. Taking into account the fact that chaotic solutions are obtained by computations, physicists ask (Cartwrite and Piro 1992; Gontar, 1997) whether chaotic solutions of the differential equations that model physical systems reflect the dynamic laws of nature represented by these equations or whether they are solely the result of an extreme sensitivity of these solutions to numerical procedures and computational errors. Theory of hypernumbers and extrafunctions provide a new mathematical technique for dealing with these and other problems of the theory of chaos considered by Gontar and Ilin (1991) and Gontar (1993; 1997). For example, the definition of chaos, as Gontar emphasizes, makes it problematic to use the apparatus of the conventional differential calculus to describe chaotic motion mathematically. Calculus with extrafunctions offers much more powerful and sophisticated means for treating such problems.

In addition, the theory of hypernumbers bestow possibilities for solution of another important scientific problem. Mathematical modeling of many phenomena in physics (as well as in chemistry and biology) often requires a physicist to operate with such functions that are not differentiable or even not continuous (or are discontinuous) at any point. For example, it may be important to find a derivative of such a function. This causes certain difficulties because methods of the classical mathematical analysis do not provide appropriate means for dealing with such situations. Consequently, new branches of mathematics appeared: the distribution theory and nonstandard analysis. For example, to make possible to utilize classical methods of analysis, in the distribution theory there are means of applying the operation of differentiation to arbitrary continuous functions. The nonstandard analysis does not have similar means but can deal with infinite numbers. In spite of all these new abilities, these theories do not give complete solutions to many problems that arise in physics. While in the distribution theory any continuous function has the derivative, this generalized derivative may be undefined at many points. Moreover, the problems of the divergence that appear in theoretical physics remain unsolved in the distribution theory as well as in nonstandard analysis.

In recent years in the works by Fisher $(1971 ; 1980)$, Rosinger (1980), Colombeau (1984; 1986), Li Bang-He and Li Ya-Qing (1985), Oberguggenberger (1986; 1987), Burgin (1987), Egorov (1990), Delcroix and Scarpalezos (1997) and others, a new theory of generalized functions has been developed. However, this development of distribution theory has been aimed at elaborating algebraic structures on the sets of distributions. Consequently, the possibilities of these theories are either included in theory of extrafunctions or they involve such weak topology that does not satisfy even the weakest separation axiom $T_{0}$. For example, theory of generalized function, which is developed by Egorov (1990) and is the most general in comparison with other extensions of distribution theory, allows getting solutions to a very vast class of differential equations. Theory of extrafunctions makes possible to obtain solutions to the same classes of equations but in a Hausdorff space, which has a much better topology. At the same time, in topology of the generalized functions from Egorov (1990) a sequence may have several different limits. This entails many problems for numerical solutions of differential equations.

In the second section of this paper, going after introduction, basic notions of the theory of hypernumbers are introduced. In this theory, spaces of real and complex numbers are extended to spaces of real and complex hypernumbers. The latter include the space of real and complex numbers, respectively, without any change. In contrast to this, nonstandard analysis (Robinson 1974) or theories of generalized functions from Egorov (1990) and Li Bang-He and Li Ya-Qing (1985), where generalized number domains are constructed, transfigure the initial number domain because between any pair of conventional numbers a universe of nonstandard, in particular, infinitely small, numbers is injected. At the same time, the space of hypernumbers does not contain infinitely small numbers, but contains only infinitely big numbers. This correlates with the opinion of physicists that there are no infinitely small numbers in physics. In addition, spaces of real and complex hypernumbers have a good (Hausdorff) topology, which implies such important property as uniqueness of the limit of a sequence. This provides a relevant base for elaboration of a differential calculus in the hypernumber universe.

In the third section of this paper, three classes of extrafunctions are considered: pointwise, compactwise, and measure-wise extrafunctions. All kinds of extrafunctions are constructed basing on topological principles. However, for each kind of extrafunctions a corresponding topology is used: the topology of pointwise convergence for pointwise extrafunctions, the topology of compact convergence for compactwise extrafunctions, and the topology of convergence with respect to some measure for measure-wise extrafunctions. This is a further development of the theory from Burgin (1990; 1995a; 2001a). For simplicity, we work here only with one argument real functions. However, it is possible to develop a similar theory for multidimensional real and complex functions.

To be able to solve physical problems by means of differential equations, the differential calculus for extrafunctions is constructed in the fourth part of this paper. It makes it possible to differentiate any function and to determine values of the generalized derivatives (which are called extraderivatives) at any point of its domain. If we take an ordinary function $f$ that has the classical derivative $f^{\prime}$, then the, so-called, extraderivative also coincides with $f^{\prime}$. In this partial case, it coincides with the generalized derivative either in the sense of the 
distribution theory (as a functional) or in the sense of the non-smooth analysis (as a function). It demonstrates that the calculus of extrafunctions is a natural extension of the classical calculus (as well as the theory of distributions). If the classical derivative $f^{\prime}$ of an ordinary function $f$ at some point $x$ does not exist, then the complete generalized derivative at the same point is not a single number, but some set of such numbers. It means that a generalized derivative is a relation on the set of real or complex numbers (or, more generally, hypernumbers).

\section{Denotations}

(1) $N$ is the set of all natural numbers

(2) $\omega$ is the sequence of all natural numbers

(3) $\varnothing$ is the empty set;

(4) $R$ is the set of all real numbers

(5) $R^{+}$is the set of all non-negative real numbers

(6) $R^{++}$is the set of all positive real numbers

(7) $R^{\omega}$ is the set of all sequences of real numbers

(8) If $a$ is a real number, then $|a|$ or $\|a\|$ denotes its absolute value or modulus

(9) If $a=\left(a_{i}\right)_{i \in \omega}$ is a sequence of real numbers, then $\alpha=\operatorname{Hn}\left(a_{i}\right)_{i \in \omega}$ is the real hypernumber determined by $a$

(10) $R_{\omega}$ is the set of all real hypernumbers;

(11) $R^{n}$ is an $n$-dimensional real space.

\section{HYPERNUMBERS}

Let $R^{\omega}=\left\{\left(a_{i}\right)_{i \in \omega} ; a_{i} \in R\right\}$ be the set of all sequences of real numbers.

Definition 2.1 For arbitrary sequences $a=\left(a_{i}\right)_{i \in \omega}$, $b=\left(b_{i}\right)_{i \in \omega} \in C^{\omega}$ :

$$
a \sim b \leftrightarrow \lim _{i \rightarrow \infty}\left|a_{i}-b_{i}\right|=0 .
$$

LEMmA 2.1 The relation $\sim$ is an equivalence.

DEFINITION 2.2 Classes of the equivalence $\sim$ are called real hypernumbers and their set is denoted by $R_{\omega}$.

Any sequence $a=\left(a_{i}\right)_{i \in \omega}$ determines a hypernumber $\alpha=\operatorname{Hn}\left(a_{i}\right)_{i \in \omega}$. Real hypernumbers are sets of equivalent sequences of real numbers like rational numbers are sets of equivalent fractions or real numbers are sets of equivalent fundamental sequences of rational numbers. Real numbers are identified with such hypernumbers that are determined by sequences in which all elements are equal.

Remark 2.1 In the universe $R_{\omega}$ of hypernumbers, the necessity in limits for sequences of real numbers is eliminated because any such sequence defines a hypernumber and if the sequence has a limit, then this limit coincides with this hypernumber, which is, in this case, a real number.

Relations on $R$ induce corresponding relations on $R^{\omega}$.

Definition 2.3 If $a, b \in R^{\omega}$, then

$$
\begin{array}{lll}
\text { (a) } & a \leq b \leftrightarrow \exists n & \forall i \geq n\left(a_{i} \leq b_{i}\right) \\
\text { (b) } & a<b \leftrightarrow \exists n & \forall i \geq n\left(a_{i}<b_{i}\right) .
\end{array}
$$

LEMMA 2.2 Relations $\leq$ and $<$ on $R^{\omega}$ are a partial order and a strict partial order, respectively.

These relations induce similar relations on $R_{\omega}$ :

Definition 2.4 If $\alpha, \beta \in C_{\omega}$, then

$$
\begin{gathered}
\alpha \leq \beta \leftrightarrow \exists a \in \alpha \exists b \in \beta(a \leq b) ; \\
\alpha<\beta \leftrightarrow(\exists a \in \alpha \exists b \in \beta(a<b)) \& \alpha \neq \beta .
\end{gathered}
$$

LEMma 2.3 Relations $\leq$ and $<$ on $R_{\omega}$ are a partial order and a strict partial order, respectively.

Proof We begin with the relation $<$. By the definition, a strict partial order is a transitive antisymmetric relation. Thus, we have to test these properties for $<$ on $R_{\omega}$. Before doing this, it is useful to remark that the condition ( $\exists a=$ $\left.\left(a_{i}\right)_{i \in \omega} \in \alpha \exists b=\left(b_{i}\right)_{i \in \omega} \in \beta(a<b)\right)$ from the definition of $<$, is equivalent to the condition $\left(\exists a=\left(a_{i}\right)_{i \in \omega} \in\right.$ $\alpha \exists b=\left(b_{i}\right)_{i \in \omega} \in \beta \exists n \forall i>n\left(a_{i}<b_{i}\right)$ because the equivalence of the sequences defining one hypernumber does not depend on any finite beginning of these sequences.

1. The relation $<$ is transitive on $R_{\omega}$.

Let $\alpha<\beta$ and $\beta<\gamma$ for some $\alpha, \beta, \gamma \in R_{\omega}$. Then by the definition of $<$, there are such sequences $a=$ $\left(a_{i}\right)_{i \in \omega} \in \alpha, \quad b=\left(b_{i}\right)_{i \in \omega}, \quad l=\left(l_{i}\right)_{i \in \omega} \in \beta, \quad$ and $\quad c=$ $\left(c_{i}\right)_{i \in \omega} \in \gamma$, for which the following conditions are valid: for some natural number $n$ if $i>n$, then $a_{i}<b_{i}$ and for some natural number $m$ if $j>m$, then $l_{j}<c_{j}$. Besides, $\alpha \neq \beta$ and $\beta \neq \gamma$. Then there are such positive numbers $k, h \in R^{++}$that $b_{i}-a_{i}>k$ for all $i>n$ and $c_{j}-l_{j}>h$ for all $j>m$.

Let $g=\min \{k, h\}$. Then there is such a natural number $p$ that $\left|b_{j}-l_{j}\right|<g$ for all $i>p$ because the sequences $b=\left(b_{i}\right)_{i \in \omega}$ and $l=\left(l_{i}\right)_{i \in \omega}$ define the same hypernumber $\beta$.

Let $q=\max \{m, n, p\}$. Then assuming $i>q$, we have: $c_{i}-a_{i}=c_{i}-b_{i}+b_{i}-a_{i}=c_{i}-l_{i}+l_{i}-b_{i}+b_{i}-$ $a_{i}=\left(c_{i}-l_{i}\right)+\left(l_{i}-b_{i}\right)+\left(b_{i}-a_{i}\right)>g-g+g=g$ because $b_{i}-a_{i}>g, c_{i}-l_{i}>g$ and $l_{j}-b_{j}>-g$. It implies by the definition of the relation $<$ that $\alpha<\gamma$. As $\alpha, \beta, \gamma$ are arbitrary elements from $R_{\omega}$, the relation $<$ is transitive.

2. The relation $<$ is antisymmetric on $R_{\omega}$.

Let us suppose that this is not true, in other words, that for some $\alpha, \beta \in R_{\omega}$, we have $\alpha<\beta$ and $\beta<\alpha$. By the definition of the relation $<, \alpha \neq \beta$ and there are such 
$a=\left(a_{i}\right)_{i \in \omega}, \quad d=\left(d_{i}\right)_{i \in \omega} \in \alpha \quad$ and $\quad b=\left(b_{i}\right)_{i \in \omega}, \quad l=$ $\left(l_{i}\right)_{i \in \omega} \in \beta$, for which $a_{i}<b_{i}$ and $l_{i}<l_{i}$ for all $i$.

As $a_{i}<b_{i}$, we have $b_{i}-a_{i}>0$ for all $i$. Consequently, $\lim _{i \rightarrow \infty}\left(b_{i}-a_{i}\right) \geq 0$. At the same time,

$$
\begin{aligned}
\lim _{i \rightarrow \infty}\left(b_{i}-a_{i}\right) & =\lim _{i \rightarrow \infty}\left(b_{i}-l_{i}+l_{i}-a_{i}\right) \\
& =\lim _{i \rightarrow \infty}\left(\left(b_{i}-l_{i}\right)+\left(l_{i}-d_{i}\right)+\left(d_{i}-a_{i}\right)\right) \\
& =\lim _{i \rightarrow \infty}\left(b_{i}-l_{i}\right)+\lim _{i \rightarrow \infty}\left(l_{i}-d_{i}\right)+\lim _{i \rightarrow \infty}\left(d_{i}-a_{i}\right) \\
& =\lim _{i \rightarrow \infty}\left(l_{i}-d_{i}\right)
\end{aligned}
$$

as $\lim _{i \rightarrow \infty}\left(b_{i}-l_{i}\right)=0$ and $\lim _{i \rightarrow \infty}\left(d_{i}-a_{i}\right)=0$. However, $\lim _{i \rightarrow \infty}\left(l_{i}-d_{i}\right) \leq 0$ because $l_{i}<d_{i}$ for all $i$. Consequently, $\lim _{i \rightarrow \infty}\left(b_{i}-a_{i}\right)=\lim _{i \rightarrow \infty}\left(l_{i}-d_{i}\right)=0$. It means, by the definition of a hypernumber, that $\alpha=\beta$. This contradicts our assumptions and proves that the relation $<$ is antisymmetric on $R_{\omega}$ by the principle of excluded middle.

What concerns the relation $\leq$, the above proof shows that it is transitive. In addition to this, it is asymmetric because by the definition of a hypernumber, we have that $\alpha \leq \alpha$ and $\alpha \leq \beta$ and $\beta \leq \alpha$ imply $\alpha=\beta$. Thus, $\leq$ is a partial order.

We can elaborate a classification of real hepernumbers. There are hypernumbers of three types: stable; infinite increasing and decreasing; and oscillating hypernumbers.

Example 2.1 An infinite growing hypernumber: $\alpha=$ $\operatorname{Hn}\left(a_{i}\right)_{i \in \omega}$, where $a_{i}=i, i=1,2, \ldots$.

Example 2.2 An infinite growing hypernumber: $\beta=$ $\operatorname{Hn}\left(b_{i}\right)_{i \in \omega}$, where $b_{i}=2^{i}, i=1,2, \ldots$

Example 2.3 A finite oscillating hypernumber: $\gamma=$ $\operatorname{Hn}\left(a_{i}\right)_{i \in \omega}$, where $a_{i}=(-1)^{i}, i=1,2, \ldots$.

Example 2.4 An infinite oscillating hypernumber: $\delta=$ $\operatorname{Hn}\left(a_{i}\right)_{i \in \omega}$, where $a_{i}=(-1)^{i} \cdot i, i=1,2, \ldots$

Example 2.5 An infinite decreasing hypernumber: $\nu=$ $\operatorname{Hn}\left(a_{i}\right)_{i \in \omega}$, where $a_{i}=-5 i, i=1,2, \ldots$.

Example 2.6 An infinite oscillating hypernumber: $\theta=$ $\operatorname{Hn}\left(a_{i}\right)_{i \in \omega}$, where $a_{i}=2^{i}+(-1)^{i} i, i=1,2, \ldots$.

Here we give exact definitions for classes of hypernumbers.

DEFINITION 2.5 A real hypernumber $\alpha$ is called finite or bounded if there is a sequence $\left(a_{i}\right)_{i \in \omega}$ such that $\alpha=$ $\operatorname{Hn}\left(a_{i}\right)_{i \in \omega}$ and for some positive real number $b,\left|b_{i}\right|<b$ for almost all $i \in \omega$.

LEMMA 2.4 The following conditions are equivalent:

- $\alpha$ is a finite real hypernumber;

- there is a sequence $\left(a_{i}\right)_{i \in \omega}$ such that $\alpha=\operatorname{Hn}\left(a_{i}\right)_{i \in \omega}$ and for some real number $b,\left|a_{i}\right|<b$ for all $i \in \omega$;
- for any sequence $\left(a_{i}\right)_{i \in \omega}$ such that $\alpha=\operatorname{Hn}\left(a_{i}\right)_{i \in \omega}$ there is a real number $b$ such that $\left|a_{i}\right|<b$ for almost all $i \in \omega$;

- for any sequence $\left(a_{i}\right)_{i \in \omega}$ such that $\alpha=\operatorname{Hn}\left(a_{i}\right)_{i \in \omega}$ there is a real number $b$ such that $\left|a_{i}\right|<b$ for all $i \in \omega$.

Definition 2.6 A real hypernumber $\alpha=\operatorname{Hn}\left(a_{i}\right)_{i \in \omega}$ is called stable if there is a sequence $\left(a_{i}\right)_{i \in \omega}$ such that $\alpha=$ $\operatorname{Hn}\left(a_{i}\right)_{i \in \omega}$ and for some real number $b, a_{i}=b$ for almost all $i \in \omega$.

For such a hypernumber, we will assume $\alpha=b$ (cf. Lemma 2.6).

LeMmA 2.5 Any stable hypernumber $\alpha=\operatorname{Hn}\left(a_{i}\right)_{i \in \omega}$ is finite.

LEMMA 2.6 The following conditions are equivalent:

- $\alpha$ is a stable real hypernumber;

- there is a sequence $\left(a_{i}\right)_{i \in \omega}$ such that $\alpha=\operatorname{Hn}\left(a_{i}\right)_{i \in \omega}$ and for some real number $b, a_{i}=b$ for all $i \in \omega$;

- for any sequence $\left(a_{i}\right)_{i \in \omega}$ such that $\alpha=\operatorname{Hn}\left(a_{i}\right)_{i \in \omega}$, there is a real number $b$ such that $a_{i}=b$ for almost all $i \in \omega$;

- for any sequence $\left(a_{i}\right)_{i \in \omega}$ such that $\alpha=\operatorname{Hn}\left(a_{i}\right)_{i \in \omega}$, there is a real number $b$ such that $a_{i}=b$ for all $i \in \omega$.

As stable real hypernumbers that correspond to different real numbers $b$ cannot be equal, we have the following result.

LEMMA 2.7 There is a one-to-one correspondence $f_{R}$ between $R$ and the subset $\mathrm{St} R_{\omega}$ of all stable hypernumbers from $R_{\omega}$, which is defined by the formula $f_{R}(a)==$ $\operatorname{Hn}\left(a_{i}\right)_{i \in \omega}$ with all $a_{i}=a$.

In what follows, we will identify stable real hypernumbers and corresponding real numbers. For example, $(8,8,8, \ldots, 8, \ldots)=8$ in $R_{\omega}$.

DEFINITION 2.7 A real hypernumber $\alpha=\operatorname{Hn}\left(a_{i}\right)_{i \in \omega}$ is called oscillating if

$$
\begin{gathered}
\exists k \in R^{++} \forall i \in \omega \exists j \in \omega \exists m \in \omega \exists n \in \omega(i<j \& j \\
\left.<m \& m<n \& a_{j}-a_{m}>k \& a_{n}-a_{m}>k\right) .
\end{gathered}
$$

DEFINITION 2.8 A real hypernumber $\alpha=\operatorname{Hn}\left(a_{i}\right)_{i \in \omega}$ is called strictly oscillating if

$$
\exists k \in R^{++} \forall i \in \omega\left(a_{i}-a_{i+1}>k \& a_{i+2}-a_{i+1}>k\right)
$$

Remark 2.2 Oscillating hypernumbers may be bounded or finite (cf. Example 2.3) and unbounded or infinite (cf. Example 2.4 and 2.6). 
DEFINITION 2.9 A real hypernumber $\alpha=\operatorname{Hn}\left(a_{i}\right)_{i \in \omega}$ is called infinite increasing if

$$
\begin{aligned}
& \left.\exists j \in \omega \forall i>j\left(a_{i+1}-a_{i}>0\right)\right) \&\left(\forall p \in R \exists i \in \omega \left(a_{i}\right.\right. \\
& \quad>p)),
\end{aligned}
$$

and infinite decreasing if

$$
\begin{aligned}
& \left.\exists j \in \omega \forall i>j\left(a_{i+1}-a_{i}>0\right)\right) \&\left(\forall p \in R \exists i \in \omega \left(a_{i}\right.\right. \\
& \quad>p)) .
\end{aligned}
$$

Proposition 2.1 Any finite real hypernumber is either a real number or an oscillating real hypernumber.

Proposition 2.2 Any infinite real hypernumber is either an infinite increasing number or an infinite decreasing number or an oscillating real hypernumber.

Remark 2.3 For complex hypernumbers, Propositions 2.1 and 2.2 are not true in general.

Remark 2.4 For an arbitrary partially ordered set $H$ and $H$-real hypernumbers, Propositions 2.1 and 2.2 are not true in general.

Proposition 2.3 If $\alpha=\operatorname{Hn}\left(a_{i}\right)_{i \in \omega}$ is a real number (an infinite increasing or infinite decreasing hypernumber), $\beta=\operatorname{Hn}\left(b_{i}\right)_{i \in \omega}$, and for almost all $i \in \omega, b_{i}$ is between $a_{i+1}$ and $a_{i}$, then $\beta$ is a real number (an infinite increasing or infinite decreasing hypernumber).

Remark 2.5 For oscillating hypernumbers, this is not always true as it is demonstrated in the following example.

Example 2.7 The condition of the Proposition 2.3 is satisfied for the pair that consists of the finite oscillating hypernumber $\alpha=\operatorname{Hn}\left(a_{i}\right)_{i \in \omega}, a_{i}=(-1)^{\mathrm{i}}, i=1,2, \ldots$ and for the real number $\beta=\operatorname{Hn}\left(b_{i}\right)_{i \in \omega}, b_{i}=0, i=1,2, \ldots$ However, $\beta$ is not an oscillating hypernumber.

Proposition 2.4 If $\alpha=\operatorname{Hn}\left(a_{i}\right)_{i \in \omega}$ is an oscillating hypernumber, $\beta=\operatorname{Hn}\left(b_{i}\right)_{i \in \omega}$, and the following condition is satisfied

$$
\forall i, j \in \omega \exists m, n \in \omega\left(a_{j}<a_{i} \rightarrow b_{m}<a_{j}<a_{i}<b_{n}\right)
$$

then $\beta$ is an oscillating hypernumber.

The set of all rational numbers is dense in the space of all real numbers. This property gives us the following result.

Lemma 2.8 Any class of equivalent $H$-sequences contains a sequence, all members of which are rational numbers.

In a similar way to spaces $R^{\omega}$ and $R_{\omega}$, it is possible to define spaces $Q^{\omega}$ and $Q_{\omega}$ for the space $Q$ of all rational numbers. Then Lemma 2.8 implies the following result.

THEOREM 2.1 For an arbitrary partially ordered set $H$, we have $Q_{H}=R_{H}$.
Theorem 2.1 means that if we take the set $Q$ and construct the set $Q_{\omega}$ of all rational hypernumbers, then we get the same set $R_{\omega}$ as when we construct $H$-real hypernumbers with the set of all real numbers $R$. In particular, when we consider ordinary sequences, we build by this process rational hypernumbers. The set $Q_{\omega}$ of all rational hypernumbers contains $R$. Thus, generating rational hypernumbers, we automatically obtain real numbers.

Nevertheless, we construct real hypernumbers from real numbers because it makes the construction more transparent and helps to understand properties and behavior of hypernumbers.

Definition 2.10 The spectrum Spec $a$ of a sequence $a=\left(a_{i}\right)_{i \in \omega}$ is the set $\left\{r \in R ; r=\lim _{C} a_{i}\right.$ and $C$ is an infinite subset of $\omega$ \}.

Example 2.8 If $a=\left(a_{i}\right)_{i \in \omega}$, where $a_{i}=i, i=1,2, \ldots$, then Spec $a=\varnothing$.

(12) If $a=\left(a_{i}\right)_{i \in \omega}$, where $a_{i}=(-1)^{\mathrm{i}}, i=1,2, \ldots$, then Spec $a=\{1,-1\}$.

(13) If $a=\left(a_{i}\right)_{i \in \omega}$, where $a_{i}=(-1) i, i=1,2, \ldots$, then Spec $a=\varnothing$.

(14) If $a=\left(a_{i}\right)_{i \in \omega}$, where $a_{i}=1 / i, i=1,2, \ldots$, then Spec $a=\{0\}$.

(15) If $a=\left(a_{i}\right)_{i \in \omega}$, where $a_{i}=\sin (i / 2) \pi, i=1,2, \ldots$, then Spec $a=\{1,0,-1\}$.

Lemma 2.9 The set Spec $a$ does not depend on the choice of an $H$-sequence $\left(a_{i}\right)_{i \in \mathrm{H}}$ from $\alpha$.

Definition 2.11 The spectrum Spec $\alpha$ of a real hypernumber $\alpha=\operatorname{Hn}\left(a_{i}\right)_{i \in \omega}$ is equal to the set $\{r \in R$; $r=\lim _{C} a_{i}$ and $C$ is an infinite subset of $\left.\omega\right\}$.

Remark 2.6 By definitions, if $a=\left(a_{i}\right)_{i \in \omega}$ and $\alpha=$ $\operatorname{Hn}\left(a_{i}\right)_{i \in \omega}$, then Spec $a=\operatorname{Spec} \alpha$.

Lemma 2.10 The set Spec $\alpha$ does not depend on the choice of the $H$-sequence $\left(a_{i}\right)_{i \in \mathbf{H}}$ from $\alpha$.

The spectrum of a hypernumber is a characteristic property, which allows one to discern different types of hypernumbers.

Proposition $2.5 \alpha=\operatorname{Hn}\left(a_{i}\right)_{i \in \omega}$ is a bounded oscillating real hypernumber if and only if Spec $\alpha$ is bounded and contains more than one element.

Corollary $2.7 \alpha=\operatorname{Hn}\left(a_{i}\right)_{i \in \omega}$ is a stable real hypernumber if and only if Spec $\alpha$ contains one element.

Proposition 2.6 Two stable real hypernumbers $\alpha$ and $\beta$ are equal if and only if Spec $\alpha=\operatorname{Spec} \beta$.

Remark 2.7 For other kinds of real hypernumbers, this is not true. For example, the real hypernumbers $\alpha=$ $\operatorname{Hn}\left(a_{i}\right)_{i \in \omega}$ with $a_{i}=(-1)^{\mathrm{i}}, \quad i=1,2, \ldots, \quad$ and $\beta=$ $\operatorname{Hn}\left(b_{i}\right)_{i \in \omega}$ with $b_{i}=(-1)^{i+1}, i=1,2, \ldots$, have the same spectrum Spec $\alpha=\operatorname{Spec} \beta=\{1,-1\}$. 


\section{EXTRAFUNCTIONS}

Here we give an advanced construction of real extrafunctions, which are built from the sequences of ordinary functions. In a more general case, extrafunctions or, more exactly, $H$-real extrafunctions are built from $H$-sequences where $H$ is some partially ordered set of finite extrafunctions, which are mappings from sets of real hypernumbers into real numbers (Burgin, 2001a).

Let us assume that we have a collection $E=\{X \subseteq R\}$ of subsets of $R$ and a system $Q=\left\{Q_{X} ; X \in E\right\}$ of linear functionals $Q_{X}$, in which the functional $Q_{X}$ is defined for some functions on the set $X$ from $E$. A model example for a linear functional is an integral, e.g. $E$ consists of all compact subsets of $R$ and $Q_{X}(f)=\int_{X} f \mathrm{~d} \mu_{X}$ where $\mu_{X}$ is the restriction on $X$ of the Lebesque measure $\mu$ on $R$. Other examples of linear functionals are such operations as sup and inf. For continuous functions and compact spaces, we can take operations max and min.

To define real extrafunctions, we consider the space $F R^{\omega}$ of all infinite sequences of real functions on $R$.

Definition 3.1 Two sequences $\left\{f_{n}(x) ; n \in \omega\right\}$ and $\left\{g_{n}(x) ; n \in \omega\right\}$ of real functions are equivalent if for any set $X \in E$, we have $\lim _{n \rightarrow \infty} Q_{X}\left(\left|f_{n}-g_{n}\right|\right)=0$.

Remark 3.1 Actually, we need such functionals $Q_{X}$ that are defined only on positive functions on the set $X$ from $E$.

Lemma 3.1 Two sequences $\left\{f_{n}(x) ; n \in \omega\right\}$ and $\left\{g_{n}(x) ; n \in \omega\right\}$ of real functions are equivalent if and only if the hypernumber $\alpha=\operatorname{Hn}\left(\mathrm{Q}_{X}\left(\left|f_{i}-g_{i}\right|\right)\right)_{i \in \omega}$ is equal to zero.

Definition 3.2 An $E, Q$-extrafunction $f$ on $R$ is a class of equivalent sequences of real functions on $R$.

$\operatorname{Ex}_{E, Q}(R)$ denotes the set of all $E, Q$-extrafunctions on $R$. If $\left\{f_{n}(x) ; n \in \omega\right\}$ is a sequence of real functions, then $f=E Q\left\{f_{n}(x) ; n \in \omega\right\}$ is an $E, Q$-extrafunction generated by this sequence, and the sequence $\left\{f_{n}(x) ; n \in \omega\right\}$ is called an approximation of $f$. $\operatorname{Ex}_{E, Q}(R)$ is a topological space, but its topology is considered elsewhere (for particular cases cf., Burgin, 2001b).

There is a natural mapping $m: F(R, R) \rightarrow \mathrm{Ex}_{E, Q}(R)$ of the set $F(R, R)$ of all real functions on $R$ into the set $\operatorname{Ex}_{E, Q}(R)$. Namely, if $f$ is a real function, then $m(f)=$ $E Q\left\{f_{n}(x) ; n \in \omega\right\}$ with $f_{n}(x)=f(x)$ for all $n \in \omega$. We denote the set of all approximations of a real function $f$ by Appr $f$. The sequence $A_{f}=\left\{f_{n}=f ; n \in \omega\right\}$ is called the standard approximation of $f$.

Remark 3.2 It is possible to consider functionals $Q_{X}$ that are defined for some class $F$ of functions on the set $X$ from $E$ (cf. Example 3.3). It gives us relative extrafunctions or $E, F, Q$-extrafunctions.

Let us consider some examples of extrafunctions.

Example 3.1 Restricted (or pointwise, Burgin, 2001a) extrafunctions, which were introduced by Burgin (1995a) are considered below.
Definition 3.3 A partial mapping $f: R \rightarrow R_{\omega}$ is called a real pointwise extrafunction.

We denote by REPF the set of all real pointwise extrafunctions.

Proposition 3.1 If $E$ consists of all points from $R$ and $Q_{x}(f)=f(x)$ for any point $x$ from $R$, then $\operatorname{Ex}_{E, Q}(R)$ is the set REPF of all restricted pointwise extrafunctions on $R$.

Example 3.2 Compactwise extrafunctions, which were introduced by Burgin (2001b) are considered below.

For any compact subset $K$ from $R^{n}$ and any two bounded on compact subsets functions $f$ and $g$, we can define the distance between $f$ and $g$ on $K$ :

$$
\operatorname{dist}_{K}(f, g)=\sup \{\|f(x)-g(x)\| ; x \in K\} .
$$

Here $\|u\|$ denotes the conventional norm of an element $u$, which the absolute value in the space $R$.

Definition 3.4 Two sequences $\left\{f_{n}(x) ; n \in \omega\right\}$ and $\left\{g_{n}(x) ; n \in \omega\right\}$ are called compactwise equivalent if $\lim _{i \rightarrow \infty} \operatorname{dist}_{K}\left(f_{n}, g_{n}\right)=0$ for any compact subset $K$ from $R$.

DEFinition 3.5 The classes of compactwise equivalent sequences are called compactwise extrafunctions.

We denote by $R E K F$ the set of all real compactwise extrafunctions.

Proposition 3.2 If $E$ consists of all compact subsets of $R^{n}$ and $Q_{B}(f)=\sup \{\|f(x)\| ; x \in B\}$ for any compact subset $B$ from $R$, then $\operatorname{Ex}_{E, Q}(R)$ is the set $R E K F$ of all real compactwise extrafunctions on $R$.

Example 3.3 Measure dependable extrafunctions, which were introduced by Burgin (2001b), contain an important suclass of measure-wise extrafunctions. It is considered below.

Let us assume that we have a collection $\mathbf{B}$ of all compact subsets of $R$ and a system $M=\left\{\mu_{X} ; X \in \mathbf{B}\right\}$ of measures $\mu_{X}$ is defined on each set $B$ from $\mathbf{B}$. For example, $\mu_{X}$ is the restriction on $X$ of the Lebesque measure $\mu$ on $R$.

Definition 3.6 Two sequences $\left\{f_{n}(x) ; n \in \omega\right\}$ and $\left\{g_{n}(x) ; n \in \omega\right\}$ of real functions that are integrable on each compact subset $B$ from $R$, are called measure equivalent if for any compact subset $B$ from $R$, we have $\lim _{i \rightarrow \infty} \int_{\mathrm{B}}\left|f_{n}(x)-g_{n}(x)\right| \mathrm{d} \mu_{X}=0$.

Definition 3.7 A measure-wise extrafunction $f$ on $R$ is a class of measure equivalent sequences of elements from $R$.

In this case, we consider only sequences of functions that are integrable on each compact subset $B$ from $R$ because our functional $Q_{B}$ is defined only for such functions.

We denote by $R E M F$ the set of all real measure-wise extrafunctions on $R$.

Proposition 3.3 If $E$ consists of all compact subsets of $R^{n}$ and $Q_{B}(f)=\int_{\mathrm{B}}\left|f_{n}(x)-g_{n}(x)\right| \mathrm{d} \mu_{X}$ for any compact subset $B$ from $R$, then $\operatorname{Ex}_{E, Q}(R)$ is the set $R E M F$ of all real measure-wise extrafunctions on $R$. 
Remark 3.3 Measure-wise extrafunctions are closely related to distributions as the following statement demonstrates.

Let $D$ be an open subset of $R$ and $D^{\prime}$ be the linear space of all distributions on $R$ (Schwartz, 1950).

Proposition 3.4 There is the linear subspace REMD of the space $R E M F$ of all real measure-wise extrafunctions on $D$, which is isomorphic to the space $D^{\prime}$.

Operations in the set $F R$ of all real functions induce corresponding operations in the space $F R^{\omega}$ of all infinite sequences of real functions on $R$.

Let $f=\left\{f_{n}(x) ; n \in \omega\right\}$ and $g=\left\{g_{n}(x) ; n \in \omega\right\}$ be elements from $F R^{\omega}$.

\section{DEFINITION 3.8}

- Operation of addition in $F R^{\omega}$ :

$$
\begin{aligned}
& f+g=\left\{h_{n}(x) ; n \in \omega\right\} \\
& \quad \text { where } h_{n}(x)=f_{n}(x)+g_{n}(x) \text { for all } n \in \omega ;
\end{aligned}
$$

- Operation of subtraction in $F R^{\omega}$ :

$$
\begin{aligned}
& f-g=\left\{h_{n}(x) ; n \in \omega\right\} \\
& \quad \text { where } h_{n}(x)=f_{n}(x)-g_{n}(x) \text { for all } n \in \omega ;
\end{aligned}
$$

- Operation of multiplication in $F R^{\omega}$ :

$$
\begin{aligned}
& f \cdot g=\left\{h_{n}(x) ; n \in \omega\right\} \\
& \quad \text { where } h_{n}(x)=f_{n}(x) \cdot g_{n}(x) \text { for all } n \in \omega .
\end{aligned}
$$

Proposition 3.5 Operations of addition and subtraction in $F R^{\omega}$ induce similar operations in $\operatorname{Ex}_{E, Q}(R)$.

Proof Let take some $E, Q$-extrafunctions $f=$ $E Q\left\{f_{n}(x) ; n \in \omega\right\}$ and $g=E Q\left\{g_{n}(x) ; n \in \omega\right\}$. We define $f+g=h$ from $\operatorname{Ex}_{E, Q}(R)$, where $h=E Q\left\{h_{n}(x) ; n \in \omega\right\}$ and $h_{n}(x)=f_{n}(x)+g_{n}(x)$ for all $n \in \omega$. To show that this is an operation in $\operatorname{Ex}_{E, Q}(R)$, it is necessary to prove that $h$ does not depend on the choice of sequences $\left\{f_{n}(x) ; n \in\right.$ $\omega\}$ and $\left\{g_{n}(x) ; n \in \omega\right\}$. To do this, let us take another sequence $\left\{l_{n}(x) ; n \in \omega\right\}$ in $f$ and show that if the $E, Q$ extrafunctions $p$ is equal to $E Q\left\{l_{n}(x)+g_{n}(x) ; n \in \omega\right\}$, then $f=p$.

By Definitions 3.1 and 3.2, $\lim _{n \rightarrow \infty} Q_{X}\left(\left|f_{n}-g_{n}\right|\right)=0$ for all $X \in E$. Consequently, $\lim _{n \rightarrow \infty} Q_{X}\left(\mid f_{n}+g_{n}\right)-\left(l_{n}+\right.$ $\left.\left.g_{n}\right) \mid\right)=\lim _{n \rightarrow \infty} Q_{X}\left(\left|f_{n}-l_{n}\right|\right)=0$ for all $X \in E$. Then by Definition 3.1, $f=p$.

If we take another sequence that represents the $E, Q-$ extrafunction $g$, a similar proof shows that the result of addition $f+g$ will be the same.

The proof for a difference of two $E, Q$-extrafunctions is similar to the proof for a sum of two $E, Q$-extrafunctions.

Proposition 3.5 is proved.
DeFinItion 3.9 A sequence $\left\{f_{n}(x) ; n \in \omega\right\}$ from $F R^{\omega}$ is called bounded if for any $X \in E$, there is a positive real number $q$ such that $\left|f_{n}(x)\right|<q$ for all $n \in \omega$ and all $x \in$ $X$.

Bounded sequences from $F R^{\omega}$ define bounded extrafunctions from $\operatorname{Ex}_{E, Q}(R)$. Let us consider the set $\mathrm{Bo} F R^{\omega}$ of all bounded sequences from $F R^{\omega}$ and the set $\operatorname{BoEx}_{E, Q}(R)$ of the real $E, Q$-extrafunctions that are defined by sequences from BoFR ${ }^{\omega}$.

LEMMA 3.2 $\mathrm{Bo} F R^{\omega}$ and $\mathrm{BoEx}_{E, Q}(R)$ are linear spaces.

In what follows, all $Q_{X}$ are positive functionals, i.e. if $f(x)>0$ for all $x \in X$, then $Q_{X}(f)>0$.

LemMA 3.3 If $f>g$ and $Q$ is a positive functional, then $Q(f)>Q(g)$.

Proposition 3.6 The operation of multiplication by elements from $\mathrm{BoEx}_{E, Q}(R)$ is defined in $\operatorname{Ex}_{E, Q}(R)$.

Proof Let us take an arbitrary $E, Q$-extrafunction $f=$ $E Q\left\{f_{n}(x) ; n \in \omega\right\} \in \operatorname{Ex}_{E, Q}(R)$ and an arbitrary bounded $E, Q$-extrafunction $g=E Q\left\{g_{n}(x) ; n \in \omega\right\} \in \operatorname{Ex}_{E, Q}(R)$. Then $f \cdot g=E Q\left\{h_{n}(x) ; n \in \omega\right\}$ where $h_{n}(x)=f_{n}(x) \cdot g_{n}(x)$ for all $n \in \omega$. To show that this is an operation in $\operatorname{Ex}_{E, Q}(R)$, it is necessary to prove that $f \cdot g=h$ does not depend on the choice of the sequences $\left\{f_{n}(x) ; n \in \omega\right\}$ and $\left\{g_{n}(x) ; n \in \omega\right\}$. To do this, let us take another sequence $\left\{l_{n}(x) ; n \in \omega\right\}$ in $f$ and show that if $k=E Q\left\{k_{n}(x) ; n \in \omega\right\}$ where $k_{n}(x)=l_{n}(x) \cdot g_{n}(x)$ for all $n \in \omega$, then $h=k$.

By Definition 3.1, for any set $X \in E$, we have $\lim _{n \rightarrow \infty} Q_{X}\left(\left|f_{n}-l_{n}\right|\right)=0$. Besides, there is a positive real number $q$ such that $\left|g_{n}(x)\right|<q$ for all $n \in \omega$ and all $x \in X$.

Consequently, $\quad \lim _{n \rightarrow \infty} Q_{X}\left(\left|f_{n} g_{n}-l_{n} g_{n}\right|\right) \leq$ $\lim _{n \rightarrow \infty} Q_{X}\left(\left|f_{n} q-l_{n} q\right|\right)$ as $Q_{X}$ is a positive functional. The number $q$ is positive. Consequently, $\left|f_{n} q-l_{n} q\right|=$ $q \cdot\left|f_{n}-l_{n}\right|$. As $Q_{X}$ is a linear functional, we have $\lim _{n \rightarrow \infty} Q_{X}\left(\left|f_{n} q-l_{n} q\right|\right)=q \cdot \lim _{n \rightarrow \infty} Q_{X}\left(\left|f_{n}-l_{n}\right|\right)=0$.

Consequently, $\lim _{n \rightarrow \infty} Q_{X}\left(\left|f_{n} g_{n}-l_{n} g_{n}\right|\right)=0$. Then by Definition 3.1, $f \cdot g=E Q\left\{l_{n}(x) \cdot g_{n}(x) ; n \in \omega\right\}$. It means that the definition of the product $f \cdot g$ does not depend on the choice of a sequence from $f$. In a similar way, we can also prove that the product $f \cdot g$ does not depend on the choice of a sequence from $g$. Consequently, multiplication by elements from the set $\operatorname{BoEx}_{E, Q}(R)$ is defined in $\operatorname{Ex}_{E, Q}(R)$.

Proposition 3.6. is proved.

Remark 3.4 By the definition of addition and multiplication in the set $\operatorname{Ex}_{E, Q}(R)$, all laws of operations with real functions (commutativity of addition, associativity of addition, commutativity of multiplication, associativity of multiplication, and distributivity) are valid for corresponding operations with real extrafunctions.

COROLlary 3.1 The operation of multiplication by elements of $R$ in $F R^{\omega}$ induces operation of multiplication by elements of $R$ in $\operatorname{Ex}_{E, Q}(R)$. 
Corollary 3.2 The set $\operatorname{BoEx}_{E, Q}(R)$ of all bounded $E, Q$-extrafunctions is a linear algebra over the field $R$ of real numbers.

Propositions 3.5 and 3.6 imply the following result.

TheOREM 3.1 The set $\operatorname{Ex}_{E, Q}(R)$ of all real $E, Q$ extrafunctions is a module over the algebra $\mathrm{BoEx}_{E, Q}(R)$.

Corollary 3.3 The set $\operatorname{Ex}_{E, Q}(R)$ of all real $E, Q$ extrafunctions is a linear infinite dimensional space over the field $R$ of real numbers.

Proposition 3.7 The natural mapping $m: F(R, R) \rightarrow$ $\operatorname{Ex}_{E, Q}(R)$ is a monomorphism of the algebra $F(R, R)$ into the linear space $\operatorname{Ex}_{E, Q}(R)$.

As the equivalence relation defining compactwise extrafunctions identifies less function than both or the equivalence relation defining pointwise and measure-wise extrafunctions, we have the following result.

\section{THEOREM 3.2}

- There is a homomorphic projection $\mathrm{p}_{\mathrm{cp}}: R E C F \rightarrow$ $R E P F$ of the linear space of all real compactwise extrafunctions $R E C F$ on the linear space $R E P F$ of all real pointwise extrafunctions.

- There is a homomorphic projection $\mathrm{p}_{\mathrm{cp}}: R E C F \rightarrow$ $R E M F$ of the linear space of all real compactwise extrafunctions $R E C F$ on the linear space $R E M F$ of all real measure-wise extrafunctions.

In what follows, we also need extrafunctions with $\omega^{2}$ as the set of indices.

As above, $E=\{X \subseteq R\}$ is a collection $E=\{X \subseteq R\}$ of subsets of $R, Q=\left\{Q_{X} ; X \in E\right\}$ is a system of linear functionals $Q_{X}$, in which the functional $Q_{X}$ is defined for all functions on the set $X$ from $E$ and $F R^{\omega 2}$ is the space of all infinite bisequences $\left\{f_{m n}(x) ; m, n \in \omega\right\}$ of real functions $f_{m n}(x)$ on $R$.

Definition 3.10 Two bisequences $\left\{f_{m n}(x) ; m, n \in \omega\right\}$ and $\left\{g_{m n}(x) ; m, n \in \omega\right\}$ of real functions are equivalent if for any set $X \in E$, we have $\lim _{n \rightarrow \infty} Q_{X}\left(\left|f_{m n}-g_{m n}\right|\right)=0$ with an arbitrary fixed $m \in \omega$ and $\lim _{m \rightarrow \infty} Q_{X}\left(\mid f_{m n}-\right.$ $\left.g_{m n} \mid\right)=0$ with an arbitrary fixed $n \in \omega$.

Definition 3.11 An $E, Q 2$-extrafunction or an $\omega^{2}$-real $E, Q$-extrafunction $f$ on $R$ is a class of equivalent bisequences of real functions on $R$.

$\operatorname{Ex}_{E, Q_{2}}(R)$ denotes the set of all $E, Q 2$-extrafunctions on $R$. If $\left\{f_{n}(x) ; n \in \omega\right\}$ is a sequence of real functions, then $f=E Q\left\{f_{n}(x) ; n \in \omega\right\}$ is an $E, Q 2$-extrafunction generated by this sequence, and the sequence $\left\{f_{n}(x) ; n \in \omega\right\}$ is called an approximation of $f$. If $f$ is a real function, then we denote the set of its approximations by Appr $f$.

There is a natural mapping $m 2: \operatorname{Ex}_{E, Q}(R) \rightarrow \operatorname{Ex}_{E, Q_{2}}(R)$. Namely, if $f=E Q\left\{f_{n}(x) ; n \in \omega\right\}$ is a real $E, Q$-extrafunction, then $m(f)=E Q\left\{f_{n m}(x) ; m, n \in \omega\right\}$ with $f_{m}(x)=f(x)$ for all $m \in \omega$. We denote the set of all approximations in of a real $E, Q$-extrafunction $f$ in $\operatorname{Ex}_{E, Q 2}(R)$ by $\operatorname{Appr} f$. The sequence $A_{f}=\left\{f_{n m}=f_{n} ; m, n \in \omega\right\}$ is called the standard approximation of $f$.

THEOREM 3.3 The set $\operatorname{Ex}_{E, Q 2}(R)$ of all real $E, Q 2$ extrafunctions is a module over the algebra $\operatorname{BoEx}_{E, Q_{2}}(R)$ of bounded real $E, Q 2$-extrafunctions.

Corollary 3.4 The set $\operatorname{Ex}_{E, Q_{2}}(R)$ of all real E,Q2extrafunctions is a linear infinite dimensional space over the field $R$ of real numbers.

As in the case of extrafunctions defined by sequences, we can consider pointwise, compactwise and measurewise extrafunctions defined by bisequences. We call them $\omega^{2}$-real pointwise, compactwise and measure-wise extrafunctions and denote their spaces by RECF $2, R E C F 2$, and $R E C F 2$, correspondingly. In this context, $\omega^{2}$-real compactwise extrafunctions are $H$-real compactwise extrafunctions and $\omega^{2}$-real pointwise extrafunctions are $H$-real pointwise extrafunctions in the sense of Burgin (2001a).

\section{THEOREM 3.4}

- There is a homomorphic projection $\mathrm{p}_{\mathrm{cp}}: R E C F 2 \rightarrow$ $R E P F 2$ of the linear space of all real compactwise bisequential extrafunctions $R E C F 2$ on the linear space $R E P F 2$ of all real pointwise bisequential extrafunctions.

- There is a homomorphic projection $\mathrm{p}_{\mathrm{cp}}: R E C F 2 \rightarrow$ $R E M F 2$ of the linear space of all real compactwise bisequential extrafunctions $R E C F 2$ on the linear space REMF2 of all real measure-wise bisequential extrafunctions.

\section{EXTRADERIVATIVES}

Let $x \in R$.

Definition 4.1 A sequence $I=\left\{\left(a_{i}, b_{i}\right) ; i \in \omega\right\}$ of pairs of real numbers (real vectors) is called an approximation of the real number (real vector) $x$ if $x=$ $\lim _{i \rightarrow \infty} a_{i}=\lim _{i \rightarrow \infty} b_{i}$.

DefINITION 4.2 An approximation $I=\left\{\left(a_{i}, b_{i}\right) ; i \in \omega\right\}$ of $x$ is called:

- two-sided if $a_{i}<x<b_{i}$ for all $i \in \omega$;

- left if $a_{i}<b_{i} \leq x$ for all $i \in \omega$;

- strict left if $a_{i}<b_{i}=x$ for all $i \in \omega$;

- right if $x \leq a_{i}<b_{i}$ for all $i \in \omega$;

- strict right if $x=a_{i}<b_{i}$ for all $i \in \omega$;

- centered if either $x=a_{i}<b_{i}$ or $a_{i}<b_{i}=x$ for all $i \in \omega$.

The sets of all (all two-sided, left, strict left, right, strict right, centered) approximations of $x$ is denoted by appr $x$ (bappr $x$, lappr $x$, slappr $x$, rappr $x$, srappr $x$, cappr $x$, respectively). 
LeMMA 4.1 appr $x=$ bappr $x \cup$ lappr $x \cup$ rappr $x$. Let $J, I \in$ apprx.

Definition 4.3 An approximation $J=\left\{\left(a_{i}, b_{i}\right) ; i \in\right.$ $H \subseteq \omega\}$ is called a subapproximation of $I=\left\{\left(a_{i}, b_{i}\right) ; i \in\right.$ $\omega\}$. It is denoted by $J \subseteq I$.

LemmA 4.2 If $J \subseteq I$ and $I \in$ bappr $x$ ( $I \in$ lappr $x$, $I \in$ rappr $x)$ then $J \in$ bapprx $\quad(J \in$ lappr $x, \quad I \in$ rappr $x$, respectively).

Let $(R \times R)^{\omega}$ denotes the set of all sequences of pairs of real numbers.

Definition 4.4 A mapping $I: R \rightarrow(R \times R)^{\omega}$ is called an $R$-approximation on $R$ if $I(x)$ is an approximation of $x$ for all $x \in R$.

To define extraderivatives for an $E, Q$-extrafunction $f=$ $E Q\left\{f_{n}(x) ; n \in \omega\right\}$, we consider an $R$-approximation $I$, the sequence $F=\left\{f_{n}(x) ; n \in \omega\right\}$ of real functions, the bisequence $\left(\Delta_{m} f_{n} / \Delta_{m} x\right)_{(m, n) \in \omega^{2}}$ of real functions, where $\Delta_{m} x=b_{m}-a_{m} \neq 0, \quad \Delta_{m} f_{n}=f_{n}\left(b_{m}\right)-f_{n}\left(a_{m}\right), \quad$ and $\left(a_{m}, b_{m}\right) \in I(x)$ for all $x \in R$ and for all $m \in \omega$.

DEFINITION 4.5 The partial extraderivative $\partial / \partial F, I f(x)$ of $f$ with respect to $F$ and to $I$ is the $E, Q 2$-extrafunctions that is defined by the bisequence $\left(\Delta_{m} f_{n} / \Delta_{m} x\right)_{(m, n) \in \omega^{2}}$ for the same $E$ and $Q$.

We can define values of extraderivatives at separate points of $R$. However, it is necessary to have in mind that while some types of extrafunctions are determined by their values at points (for example, pointwise extrafunctions, Burgin, 1995a), other types of extrafunctions are not determined by their values at points (for example, compactwise extrafunctions, Burgin, 2001a).

Definition 4.6 The value at $a$ of the partial extraderivative $\partial / \partial F, I f(a)$ of $f$ at $a$ with respect to $F$ and to $I \in$ appra is equal to the hypernumber $\operatorname{Hn}\left(\Delta_{m} f_{n} / \Delta_{m} x\right)_{(m, n) \in \omega^{2}}$ where $\Delta_{m} x=b_{m}-a_{m}, \Delta_{m} f_{n}=$ $f_{n}\left(b_{m}\right)-f_{n}\left(a_{m}\right)$, and $\left(a_{m}, b_{m}\right) \in I$ for all $m$.

Remark 4.1 Taking some of the classes of approximations, which are defined above, we obtain corresponding types of partial extraderivatives, for example, strict right or centered partial extraderivative.

Let us build an extraderivative for an arbitrary real function $f$. To do this, we utilize the standard approximation $A_{f}=\left\{f_{n}=f ; n \in \omega\right\}$ of $f$.

DEFINITION 4.7 The partial extraderivative $\partial /$ a $f(x)$ of $f$ with respect to $I$ is the $E, Q$-extrafunctions that is defined by the sequence $\left(\Delta_{m} f / \Delta_{m} x\right)_{m \in \omega}$ for the same $E$ and $Q$.

In the same way as for extrafunctions, we can define extraderivatives of a real function $f$ at separate points of $R$.

Definition 4.8 The value at $a$ of the partial extraderivative $\partial /{ }_{\partial I} f(a)$ at a point $a$ with respect to an approximation $I$ is equal to the hypernumber $\operatorname{Hn}\left(\Delta_{m} f / \Delta_{m} x\right)_{m \in \omega}$ where $\Delta_{m} x=b_{m}-a_{m}, \Delta_{m} f=f\left(b_{m}\right)$ $f\left(a_{m}\right)$, and $\left(a_{m}, b_{m}\right) \in I$ for all $m$.
Remark 4.2 $\partial /{ }_{\partial I} f_{x}=\partial / \partial A, I f_{x}$ where $A=\left\{f_{n} ; n \in \omega\right\}$ and all $f_{n}=f$.

For example, let us take the function $f(x)=|x|$. Its extraderivative $\partial /{ }_{\partial I} f(0)$ at 0 may be equal to any number from the interval $[-1,1]$. Besides, the value of $\partial / \partial I f(0)$ may be equal to some hypernumbers (such as $\operatorname{Hn}\left(a_{i}\right)_{i \in \omega}$ with $a_{2 k}=1, a_{i 2 k-1}=-1$, and $\left.k=1,2, \ldots\right)$.

DeFinition 4.9 An extraderivative $\partial /{ }_{\partial I} f(a)$ is called one-sided (two-sided) if $I \in$ rappr $x \cup$ lapprx $(I \in$ bappr $x$ ).

The construction of the partial extraderivative $\partial /{ }_{\text {д }} f(a)$ at a point $a$ with respect to an approximation $I$ represents procedure of computation for derivatives of real functions. Really (Burden and Faires, 2001), there are different kinds of formulas for such computations. If we compare these formulas to extraderivatives, we can see that the forwarddifference formula for computation corresponds to strict right approximations and strict right extraderivatives, the backward-difference formula for computation corresponds to strict left approximations and strict left extraderivatives, and the three-point formula for computation corresponds to two-sided approximations and twosided extraderivatives. Other computational formulas for derivatives induce new constructions of extraderivatives.

The difference between classical approach and the construction introduced here is that in the classical derivative computation, it is assumed that the result does not depend on the choice of points and procedures because there is only one (if any) value for the classical derivative. At the same time, an extraderivative essentially depends on initial data and computational procedures. Taking points closer and closer to some point $a$, we approach the value of the classical derivative of $f$ the point $a$ if it exists. When the classical derivative of $f$ at $a$ does not exist, we obtain a new generalized concept of the derivative of $f$ at a point $a$, which is applicable to a much larger universe of functions.

Being closer to computational procedures, than the classical derivative extraderivative may be even more enhanced if it will take into account imprecision of computation. A technique to do this is based on neoclassical analysis, and namely on such concepts as fuzzy continuous functions (Burgin, 1995b) and fuzzy limits (Burgin, 2000a). In this more general context, difference operators become particular cases of differential operators and difference equations become particular cases of differential equations.

Let $a, b \in R, F \in A p p r f, G \in A p p r g$, and $H=a F+$ $b G$. Then Theorem 3.3 and Propositions 3.5 and 3.6 imply the following result.

THEOREM $4.1 \quad \partial /{ }_{\partial H, I}(a f+b g)(x)=a(\partial / \partial F, I f(x))+$ $b(\partial / \partial G, I g(x))$ for an arbitrary $R$-approximation $I$.

Let $a, b \in R$ and $f, g$ be some real functions. Then Theorem 4.1 implies the following result.

COROLLARY 4.1 $\partial /{ }_{\partial I}(a f+b g)=a \cdot \partial / \partial I f+b \cdot \partial / \partial I g$. 
Extraderivatives are natural extensions of conventional derivatives of functions. For simplicity, we show this only for pointwise extrafunctions.

Let $f$ be an arbitrary extrafunction, $F=\left\{f_{n}, n \in \omega\right\} \in$ $f$, and $T=\left\{I_{\theta} \in \operatorname{appr} x ; \theta \in \mathrm{A}\right\}$.

Definition 4.10 An extrafunction $f$ is called:

- $T$-differentiable at a point $x$ with respect to $F$ if for any $I, J \in T$, we have $\partial /_{\partial F, I} f(x)=\partial /_{\partial F, J} f(x)$, or equivalently, $\partial /_{\partial F, T} f(x)$ consists of a single element;

- $a$-differentiable at a point $x$ with respect to $F$ if for any $I, J \in \operatorname{appr} x$, we have $\partial / \partial F, I f(x)=\partial / \partial F, J f(x)$, or equivalently, $\partial / \partial F, \operatorname{appr} x f(x)$ consists of a single element;

- $W$-differentiable at a point $x$ with respect to $I$ if for any $F, G \in W \subseteq A p p r f$, we have $\partial /_{\partial F, I} f(x)=\partial /_{\partial G, I} f(x)$;

- completely $W$-differentiable at a point $x$ if for any $I, J \in \operatorname{appr} x$ and for any $F, G \in W \subseteq \operatorname{Appr} f$, we have $\partial / \partial F, I f(x)=\partial / \partial G, J f(x)$

- $R$-differentiable at $x$ with respect to an approximation $I \in \operatorname{appr} x$ and to an approximation $F \in \operatorname{Appr} f$ if all hypernumbers $\partial / \partial F, I f(x)=\operatorname{Hn}\left(\Delta m f n / \Delta_{m} x\right)_{(m, n) \in \omega^{2}}$ are real numbers.

THEOREM 4.2 The following conditions are equivalent:

- $f$ is $a$-differentiable at $x$ with respect to $F$;

- $f$ is $R$-differentiable and $a$-differentiable at $x$ with respect to $F$ and to any $I \in \operatorname{appr} x$;

- all functions $f_{n} \in F$ have classical derivatives at $x$.

Proof $\mathrm{b}$ implies a because conditions from $\mathrm{b}$ include conditions from a.

$\mathrm{c}$ implies b: Really, if all functions $f_{n}$ are differentiable (condition c), then by the definition of the classical derivative at a point, for any $I, J \in \operatorname{appr} x$, we have $\partial / \partial F, I f(x)=\partial /_{\partial F, I} f(x)$ (condition $\mathrm{b}$ ).

a implies c: Let us suppose that condition $\mathrm{c}$ is not true. It means that for some $f_{n}$ does not have the classical derivative at $x$. Then either two sequences $\left(f_{n}\left(b_{i}\right)-\right.$ $\left.f_{n}\left(a_{i}\right)\right) /\left(b_{i}-a_{i}\right)$ and $\left(f_{n}\left(c_{i}\right)-f_{n}\left(d_{i}\right)\right) /\left(c_{i}-d_{i}\right)$ have different limits, or there is such a sequence $\left(f_{n}\left(b_{i}\right)-\right.$ $\left.f_{n}\left(a_{i}\right)\right) /\left(b_{i}-a_{i}\right)$, which diverges.

In the first case, we take the two sequences of pairs $I=\left\{\left(b_{i}, a_{i}\right) ; i \in \omega\right\}$ and $J=\left\{\left(c_{i}, d_{i}\right) ; i \in \omega\right\}$. They are approximations of $x$. Then by Definition 6.5, for these approximations $I$ and $J$ from the set apprx, we have $\partial / \partial F, I f(x) \neq \partial / \partial F, J f(x)$. Consequently, condition a is also invalid.

In the second case, we take the sequence of pairs $I=$ $\left\{\left(b_{i}, a_{i}\right) ; i \in \omega\right\}$. Each pair $\left(b_{i}, a_{i}\right)$ corresponds to the ratio $\left(f_{n}\left(b_{i}\right)-f_{n}\left(a_{i}\right)\right) /\left(b_{i}-a_{i}\right)$. Because, by our assumption, the sequence $\left(\left(f_{n}\left(b_{i}\right)-f_{n}\left(a_{i}\right)\right) /\left(b_{i}-a_{i}\right)\right)_{i \in \omega}$ diverges, it defines the infinite hypernumber $\operatorname{Hn}\left(\left(f_{n}\left(b_{i}\right)-\right.\right.$ $\left.\left.f_{n}\left(a_{i}\right)\right) /\left(b_{i}-a_{i}\right)\right)_{i \in \omega}$.

If we have some diverging sequence $\left(l_{i}\right)_{i \in \omega}$, then we can find such its subsequence $\left(r_{i}\right)_{i \in \omega}$ that it diverges essentially faster then the initial sequence, i.e. the ratio $r_{i} / l_{i}$ tends to infinity. Consequently, by the definition of a hypernumber, the hypernumbers $\operatorname{Hn}\left(l_{i}\right)_{i \in \omega}$ and $\operatorname{Hn}\left(r_{i}\right)_{i \in \omega}$ are different.

Let us consider the sequence $\left(\left(f_{n}\left(b_{i}\right)-\right.\right.$ $\left.\left.f_{n}\left(a_{i}\right)\right) /\left(b_{i} a_{i}\right)\right)_{i \in \omega}$ as the diverging sequence $\left(l_{i}\right)_{i \in \omega}$. Then it contains a faster diverging subsequence $\left(r_{i}\right)_{i \in \omega}$. Taking those pairs from the sequence $I$, which correspond to this subsequence $\left(r_{i}\right)_{i \in \omega}$, we get another approximation $J$ of the point $x$. For this approximation, we have $\partial /{ }_{\partial F, I} f(x) \neq$ $\partial / \partial F, I f(x)$ because $\operatorname{Hn}\left(l_{i}\right)_{i \in \omega} \neq \operatorname{Hn}\left(r_{i}\right)_{i \in \omega}$. This invalidates the condition a.

It means that if $\mathrm{c}$ is false, then a is false. The principle of excluded middle implies that a implies $\mathrm{c}$.

Thus, we have: a implies c, c implies b, and b implies a. Consequently, they are all equivalent.

Theorem 4.2 is proved.

Let $X \subseteq R$.

Corollary 4.2 The following conditions are equivalent:

- $f$ is $a$-differentiable at all points $x$ from $X$ with respect to $F$;

- $f$ is $R$-differentiable and $a$-differentiable at all points $x$ from $X$ with respect to $F$ and to any $I \in$ appr $x$;

- all functions $f_{n} \in F$ are differentiable on $X$.

Remark 4.3 There are no completely (Apprf)-differentiable extrafunctions.

Let $f$ be a real function and $\mathrm{A}_{f}=\left\{f_{n}=f\right\}$ be its stable approximation.

Definition 4.11 A function $f$ is called:

- $T$-differential at a point $x$ if for any $I, J \in T$ we have $\partial / \partial f f(x)=\partial /{ }_{\partial J} f(x)$, or equivalently, $\partial{ }_{\partial T} f(x)$ consists of a single element;

- $a$-differentiable at a point $x$ if for any approximations $I, J \in$ appr $x$, we have $\partial /{ }_{\partial I} f(x)=\partial /{ }_{\partial J} f(x)$, or equiva-

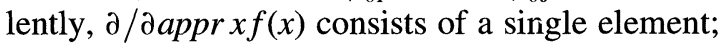

- $R$-differentiable at a point $x$ with respect to an approximation $I \in \operatorname{appr} x$ if all hypernumbers $\operatorname{Hn}\left(\Delta_{m} f / \Delta_{m} \mathrm{x}\right)_{m \in \omega}$ are real numbers.

If we take a real function $f$, its stable approximation $\mathrm{A}_{f}=\left\{f_{n}=f\right\}$ and apply Theorem 4.2, then we obtain the following result, which connects classical differentiation with the introduced here generalized differentiation.

THEOREM 4.3 The following conditions are equivalent:

- $f$ has the classical derivative $g^{\prime}$ at a point $x$ of $R$;

- $f$ is $a$-differentiable at $x$ with respect to $A_{\mathrm{f}}$;

- $f$ is $R$-differentiable at $x$ with respect to $A_{\mathrm{f}}$ and $\partial / \partial A, I f(x)=\partial / \partial I f(x)=g^{\prime}(x)$ for all $I \in$ appr $x$.

Theorem 4.3 implies that if a function has a derivative at a point $x$ of $R$ in the classical sense, then its partial extraderivative at $x$ is unique and coincides with the 
classical derivative of $f$ at $x$. Moreover, if the partial extraderivative at the point $x$ is unique, then it coincides with the classical one and, as a consequence, the function that has extraderivative is differentiable at $x$ in a conventional sense.

Let $X \subseteq R$.

COROLlaRY 4.3 The following conditions are equivalent:

- $f$ is $a$-differentiable at all points $x$ from $X$ with respect to $A_{f}$;

- $f$ is $R$-differentiable at all points $x$ from $X$ with respect to $A_{f}$ and to any $I \in \operatorname{apprx}$;

- the function $f \in F$ is differentiable on $X$.

Corollary 4.3 implies that if a function is differentiable on $X$ in the classical sense, then all its partial extraderivatives on $X$ are unique and coincide with the classical derivatives of $f$ on $X$. Moreover, if all partial extraderivatives of a function $f$ on $X$ are unique, then they coincide with the classical derivatives of $f$ on $X$ and, as a consequences, the function $f$ is differentiable on $X$ in a conventional sense.

\section{CONCLUSION}

In the present work, a new approach to analysis is presented. It is based on an extension of real numbers to a much broader universe of real hypernumbers. In addition to real numbers, this universe contains different infinite and oscillating numbers. The theory of hypernumbers is the base of the theory of extrafunctions. The results of this paper contribute to the further development of this theory, unifying results from Burgin $(1990 ; 1995 a ; 2001 a)$ and directing them to the problems of mathematical and computational physics with an emphasis on the theory of chaos.

In addition to this, the theory of extrafunctions provides new possibilities in different areas. For example, divergent processes are at the core of the classical and modern mathematical analysis. Careful control and management of these processes are essential (Bray and Stanojevic, 1998). In the universe of hypernumbers, all sequences and series of real and complex numbers as well as definite integrals of continuous functions have values. For convergent sequences, series and integrals, these values are ordinary numbers, while for divergent sequences, series and integrals, these values are infinite and oscillating hypernumbers. As a consequence, control and management of divergent processes becomes much simpler.

Problems of divergence frequently emerge in different computational problems (cf., for example, Steffen and Ingolfsdottir, 1994). Consequently, the theory of extrafunctions opens new perspectives in this direction.
One can think of the further development of the theory of extrafunctions in different directions. First, similar technique allows one to construct complex extrafunctions and to develop analysis in this context for mappings of multidimensional linear spaces.

Second, it looks beneficial to apply this theory to the problems of differential and difference equations. Specific properties of the theory of extrafunctions make it possible to achieve much more in this direction than by means of the theory of distributions or classical analysis. For example, it is possible to consider differential equations with discrete functions. In addition, this new approach correlates with computational methods of differential and difference equations solution.

Third, it would be advantageous to use the theory of extrafunctions for the development of the theory of operators and operator algebras in infinite dimensional spaces. Operator algebras are frequently used in theories of quantum fields, theory of chaos, and synergetics (cf., for example, Haag and Kastler, 1964; Horuzhy, 1986; Prigogine, 1980). Hypernumbers allow one to define hypernorms on topological spaces and algebras. With respect to operators, this makes possible to consider unbounded operators to a full extent and to study hypernormed algebras of such operators. The crucial point in this direction is the definition of multiplication of hypernumbers.

\section{References}

Bang-He, Li and Ya-Qing, Li (1985) "Non-standard analysis and multiplication of distributions in any dimension", Sci. Sin., Ser. A 28(7), 716-726.

Bray, W.O. and Stanojevic, C.V. (1998) Analysis of Divergent Control and Management of Divergent Processes (Birkhäuser, Basel).

Burden, R.L. and Faires, J.D. (2001) Numerical Analysis (Brooks/Cole, Australia/Canada/USA)

Burgin, M.S. (1987) "Algebraic structures of distributions", Doklady Acad. Sci. Ukraine 7, 5-9, (in Russian and Ukrainian).

Burgin, M.S. (1990) "Hypermeasures and hyperintegration", Doklady Natl Acad. Sci. Ukraine 6, 10-13, (in Russian and Ukrainian).

Burgin, M.S. (1995a) "Integral calculus for extrafunctions", Doklady Natl Acad. Sci. Ukraine 11, 14-17.

Burgin, M.S. (1995b) "Neoclassical analysis: fuzzy continuity and convergence", Fuzzy Sets Syst. 75(2), 291-299.

Burgin, M. (2000a) "Theory of fuzzy limits", Fuzzy Sets Syst. 115(3), 433-443.

Burgin, M.S. (2000b) "Theory of hypernumbers and Feynman integrals", Am. Math. Soc. 21(1), 71, (Abstracts).

Burgin, M. (2001a) "Extrafunctions, distributions, and nonsmooth analysis," University of California, Los Angeles, Mathematics Report Series, MRS Report 01-02, 2001, p. 47.

Burgin, M.S. (2001b) "Measure dependable extrafunctions", Am. Math. Soc. 22, 4, Abstracts.

Cartwrite, J.H.E. and Piro, O. (1992) "The dynamics of Runge-Kutt methods", Int. J. Bifurcation Chaos 2(3), 427-450.

Collins, J.C. (1984) Renormalization (Cambridge University Press, Cambridge).

Colombeau, J.-F. (1984) New Generalized Functions and Multiplication of Distributions (North Holland, Amsterdam).

Colombeau, J.-F. (1986) A Mathematical Analysis Adapted to the Multiplication of Distributions (Springer, New York).

Delcroix, A. and Scarpalezos, D. (1997) "Asymptotic scales, asymptotic algebras", Proceedings of the International Conference on Generalized Functions.

Edzawa, H., Zuneto, T. (1977) In: Quantum Physics Perspectives (Iwanami Shoten, Tokio) 
Egorov, Yu.V. (1990) "A contribution to the theory of generalized functions", Russian Math. Surveys 45(5), 1-49.

Fisher, B. (1971) "The product of distributions", Quart. J. Math. Oxford 22, 258-291.

Fisher, B. (1980) "On defining the product of distributions", Math. Nachr. 99, 239-249.

Gontar, V. (1993) "New theoretical approach for physicochemical reactions dynamics with chaotic behavior", Chaos in Chemistry and Biochemistry (World Scientific, London), pp 225-247.

Gontar, V. (1997) "Theoretical foundation for the discrete dynamics of physicochemical systems: chaos, self-organization, time and space in complex systems", Discrete Dyn. Nat. Soc. 1(1), 31-43.

Gontar, V. and Ilin, I. (1991) "New mathematical model of physicochemical dynamics", Contrib. Plasma Phys. 31(6), 681-690.

Haag, R. and Kastler, D. (1964) "An algebraic approach to quantum field theory", J. Math. Phys. 5(7), 848-861.
Horuzhy, S.S. (1986) Introduction to the Algebraic Quantum Field Theory (Nauka, Moscow), (in Russian).

Oberguggenberger, M. (1986) "Products of distributions", J. Reine Angew. Math. 36S, 1-11.

Oberguggenberger, M. (1987) "Generalized solutions to semi-linear hyperbolic systems", Monatsch. Math. 103, 133-144.

Prigogine, I. (1980) Form Being to Becoming: Time and Complexity in the Physical Systems (Freeman and Co., San Fransisco).

Robinson, A. (1974) Non-Standard Analysis (North-Holland, New York).

Rosinger, E.E. (1980) Non-Linear Partial Differential Equations (North Holland, Amsterdam).

Steffen, B. and Ingolfsdottir, A. (1994) "Characteristic Formulae for Processes with Divergence", Inform. Comput. 110(1), 149-163.

Schwartz, L. (1950-1951) Theorie de distributions (Hermann, Paris) Vol. I-II. 


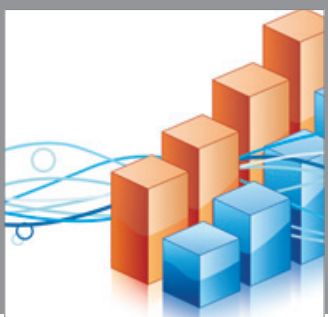

Advances in

Operations Research

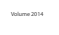

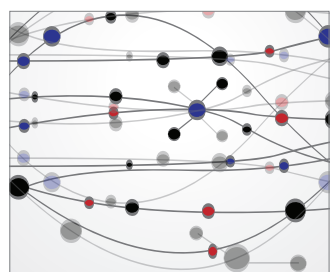

\section{The Scientific} World Journal
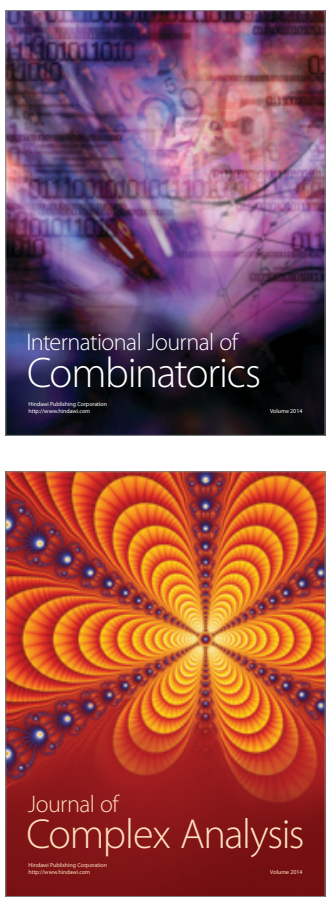

International Journal of

Mathematics and

Mathematical

Sciences
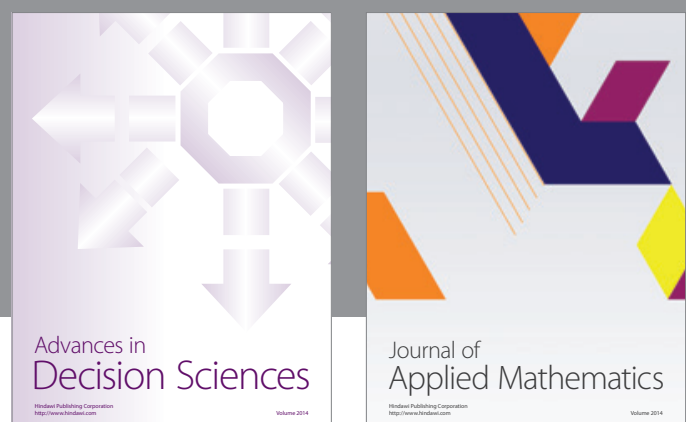

Journal of

Applied Mathematics
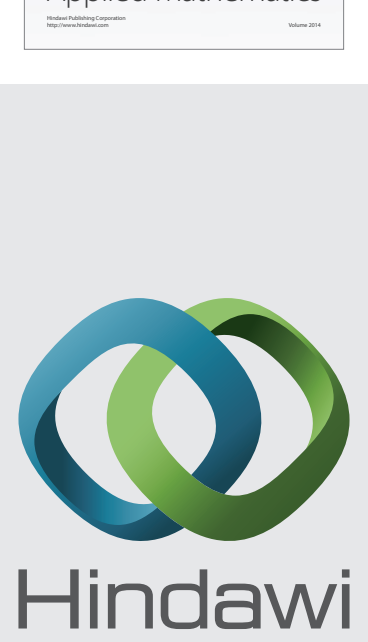

Submit your manuscripts at http://www.hindawi.com
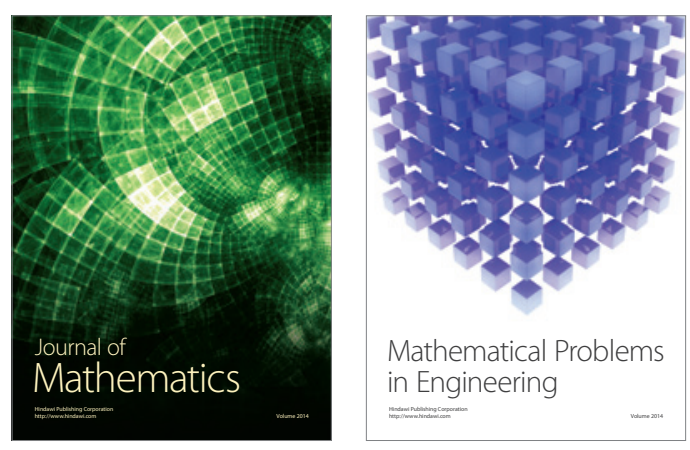

Mathematical Problems in Engineering
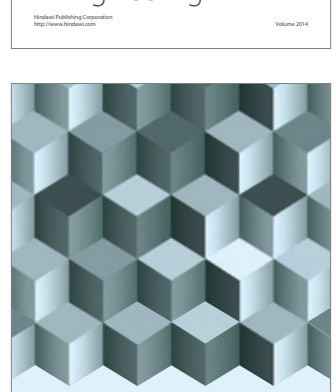

Journal of

Function Spaces
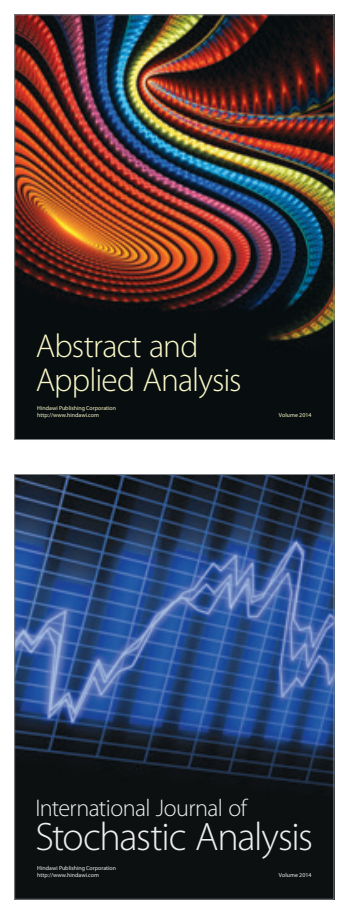

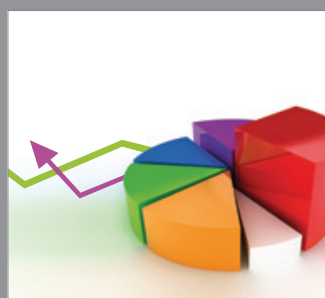

ournal of

Probability and Statistics

Promensencen
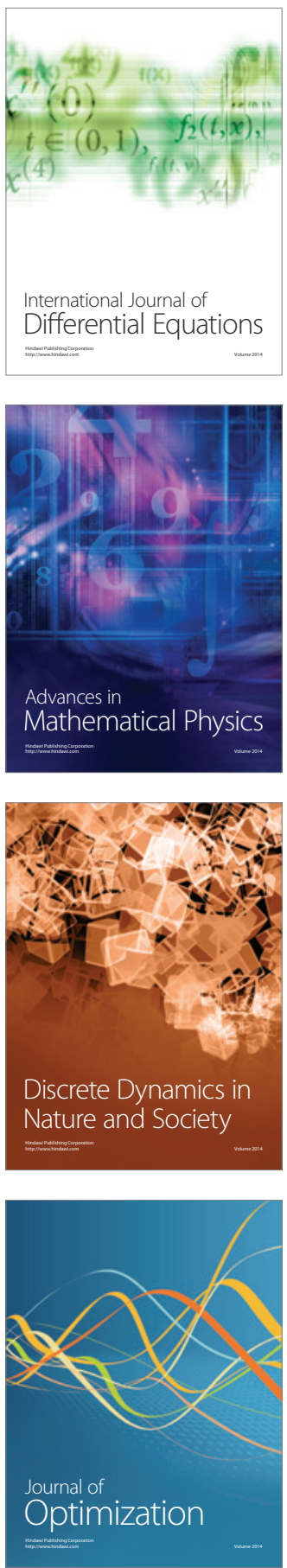